\title{
NBI-driven Alfvénic modes at ASDEX Upgrade
}

\author{
Ph. Lauber ${ }^{1}$, I.G.J. Classen ${ }^{2}$, D. Curran ${ }^{3}$, V. Igochine ${ }^{1}$, \\ B. Geiger ${ }^{1}$, S. da Graça ${ }^{4}$, M. García-Muñoz ${ }^{1}$, M. Maraschek ${ }^{1}$, \\ P. McCarthy ${ }^{4}$ and the ASDEX Upgrade Team \\ ${ }^{1}$ Max-Planck-Institut für Plasmaphysik, EURATOM-Association, Garching, \\ Germany; email: pwl@ipp.mpg.de \\ ${ }^{2}$ FOM Institute Differ - Dutch Institute for Fundamental Energy Research, \\ Association EURATOM-FOM, 3430 BE Nieuwegein, The Netherlands \\ ${ }^{3}$ Department of Physics, University College Cork, EURATOM-Association DCU, \\ Cork, Ireland \\ ${ }^{4}$ Associação EURATOM/IST, Instituto de Plasmas e Fusão Nuclear -Laboratorio \\ Associado, Instituto Superior Tecnico, 1049-001 Lisboa, Portugal
}

\begin{abstract}
A large variety of electromagnetic modes excited by NBI-generated energetic ions are observed in the early phase of many discharges at ASDEX Upgrade. In addition to the well known reversed shear Alfvén eigenmodes (RSAE) and the toroidal Alfvén eigenmodes (TAE), a set of modes around $70 \mathrm{kHz}$ is observed as recently described in [7]. The modes were identified to be beta-induced Alfvén eigenmodes (BAE) connected with the appearance of the $q=2$ and the $q=1.5$ surface during the current ramp-up phase. In the view of ITER, these BAEs may occur in scenarios with $q \approx 2$ (scenario 4) and therefore add significantly to the transport of energetic ions due to RSAEs and TAEs.

Experimentally, the combination of ECE, Soft-X-ray and magnetic measurements allows for a very reliable mode position and mode structure determination. The measurements are compared with linear gyrokinetic calculations employing the LIGKA code that uses a fully kinetic model to describe fast-particle-driven modes in general tokamak geometry .
\end{abstract}




\section{Introduction}

Alfvénic instabilities driven by energetic neutral beam ions are a very important branch of fusion research. Not only their effect on the unfavorable redistribution of energetic ions in present tokamaks has to be understood and extrapolated to the International Tokamak Experimental Reactor (ITER) [1] but also their potential for Alfvén spectroscopy[2, 3, 4], i.e. the determination of plasma parameters via the frequency evolution of these modes has to be examined.

Recently, reversed shear Alfvén eigenmodes (RSAE) [5] attracted a lot of attention. Due to advances in both the experimental measurements and the code simulation capabilities, refined information about their $2 \mathrm{~d}$ mode structures [6, 7] and transition to TAEs [8] could be obtained. Furthermore, the radial harmonics of RSAEs can be used to constrain the curvature of the q-profile at $q_{\min }$ [7] or allow to determine the GAM frequency [9]. Finally, also the non-linear interaction with other Alfvénic instabilities, such as TAEs has been investigated[10].

In this paper, we aim to describe and model another, closely connected Alfvénic mode in the same frequency regime as the RSAE, the beam-driven beta induced Alfvén eigenmode (BAE) [11, 12, 13, 16, 17, 18, 19]. Although a lot of literature is available for BAE modes they have so far not been investigated in the current ramp-up phase of a tokamak discharge. Whereas in the ramp-up phase at JET [20, 21] and DIIID [22] energetic particle driven geodesic acoustic modes (EGAMs) are observed regularly, at ASDEX Upgrade electromagnetic modes with finite toroidal mode numbers $n=1,2$ are detected. It will be shown, that the modes are not fishbones [15, 14]. Moreover, steady state modes and bursting modes as well as transitions between these two states can be observed.

Whereas some of the experimental properties of the BAE modes were already described in ref. [7], this paper focuses on the theoretical description of the observed BAE modes and in particular on their existence conditions, their damping and their drive by isotropic and anisotropic energetic particle distribution functions.

In the first part, the experimental observations and the equilibrium parameters used for the numerical investigations are presented. In the second part, the theoretical model, the LIGKA code [23] is briefly described. In the third part, both local and global results concerning the properties of the BAE modes are shown. Finally, conclusions and an outlook to further experimental and theoretical work are given.

\section{Experimental observations}

2.5 MW of NBI heating (93 keV beam) was applied in the current ramp-up phase of a series of low density discharges, identical to the scenarios described in $[24,25,26]$. The magnetic field was $2 T$. The time traces of the line integrated electron density $n_{e}$ as well as electron temperature $T_{e}$ can be found in ref [7]. For convenience, these traces are shown in the appendix, fig 9 . As the current penetrates towards the magnetic 


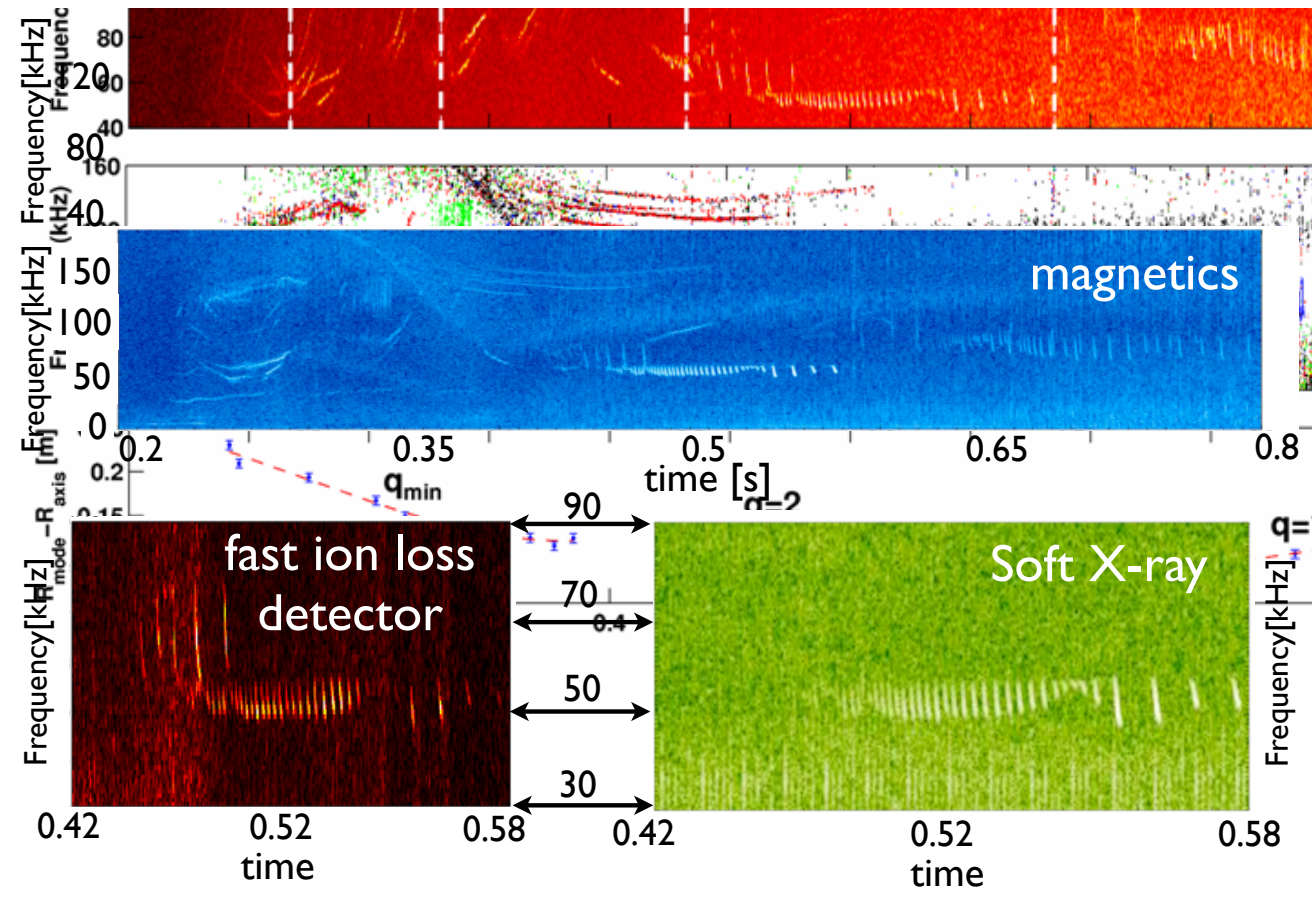

Figure 1. spectrograms from ECEI (top), magnetic pick-up coils (middle), fast ion loss detector (bottom left) and central SXR channel I53 (bottom right)

axis, a reversed q-profile is established. Although no MSE data was available for this discharge, the value of $q_{\min }$ as a function of time and its radial position can be inferred from the evolution of the RSAEs as described for the discharge in ref. [7]. As soon as the $q=2$ surface is crossed, a strong mode activity around $60 \mathrm{kHz}$ is observed. It is clearly visible in the magnetic pick-up coils, in the central soft-X-ray channels (SXR-J_51) [27], the ECEI [28] and in the fast-ion loss detector (FILD) [29, 30]. In fact, the losses between $t=0.44-0.58 \mathrm{~s}$ are the only losses detected in this discharge. This observation underlines the importance of understanding the properties of this very strongly redistributing mode. Analysing the phase information of the pick-up coils shows that the mode whose frequency starts to chirp at $55 \mathrm{kHz}$ has a toroidal mode number $n=1$ whereas the mode at $75 \mathrm{kHz}$ has $n=2$. The bursting mode at the later time point $t=0.63$ is related to the crossing of the $q=1.5$ surface and has $n=2$. The modes' radial and poloidal structure are shown in fig 2 . Both modes are localised around $R=1.80 \mathrm{~m}$, in terms of the square root of the normalised poloidal flux this is $\varrho_{p o l}=0.4$. Here, $\varrho_{p o l}$ is the square root of the normalised poloidal flux. Figure 2 shows clearly that they both $(n=1: \mathrm{b}, \mathrm{c} ; n=2$ : d,e $)$ move radially outwards with time. This radial motion takes place on the time scale of the q-profile relaxation, i.e. due to the movement of the $q=2$ surface. The radial mode widths are $8-10 \mathrm{~cm}$ and the poloidal mode numbers determined from the ECEI data are $m=2$ for the $n=1$ mode and $m=4$ for the $n=2$ mode. 


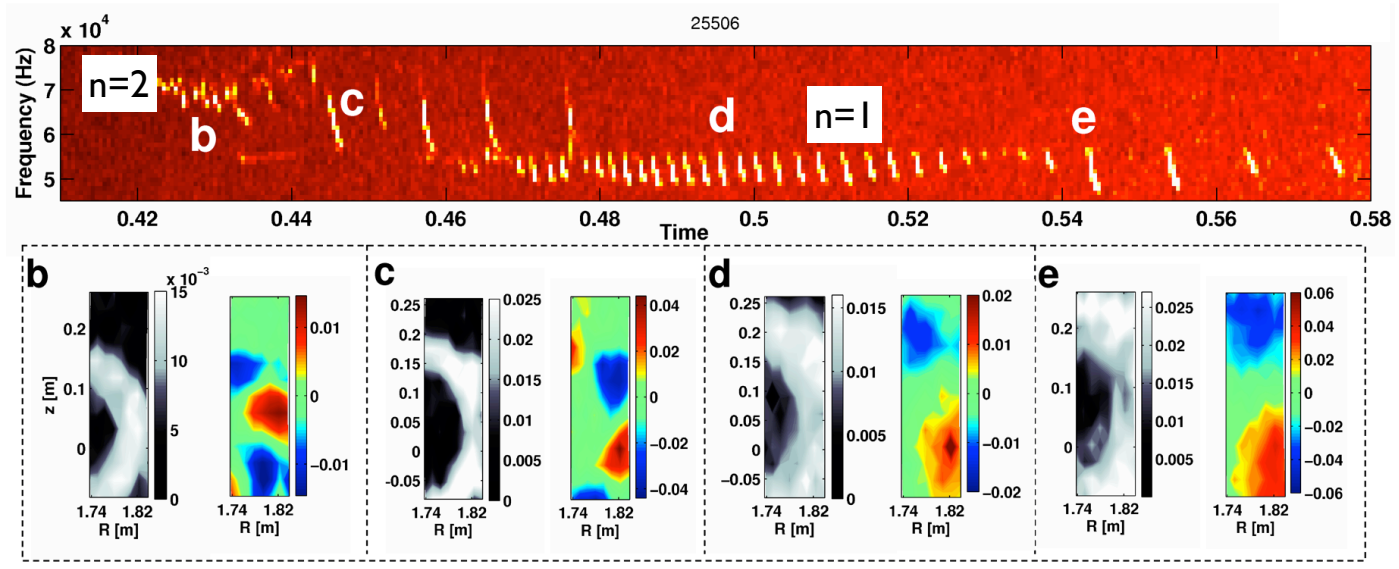

Figure 2. 2D ECEI mode structure of the $n=1$ (at $55 \mathrm{kHz}$ ) and the $n=2$ (at 75 $\mathrm{kHz}$ ) BAE modes at different time points. The black and white figures show the $2 \mathrm{D}$ ECE amplitude $A$ whereas the colored plots give the 2D phase information $(A \cos \varphi)$.

\section{Theoretical Model}

Although it is well known that the transport of energetic particles due to resonant mode interaction processes is essentially non-linear, a linear analysis is an important intermediate step due to the following reasons: first, it allows to identify the nature of the modes via analysing the frequency scaling. Secondly, the damping, the drive and stability boundaries can be investigated and finally the mode structure can be obtained. All these properties can be compared to the experiment and in the recent years it has been shown that (gyro)-kinetic models rather than MHD or hybrid MHD models are necessary to capture the properties of Alfvénic modes, especially when their frequencies are in the intermediate regime between the Alfvén frequency, the sound frequency and the diamagnetic frequency.

In this frequency range, the background ions start to influence not only the damping rate but also the mode frequency. The lower the frequency, the more important is also the influence of trapped background ions $[18,31]$. In this paper no attempt to model the non-linear phase of the mode evolution is made. However, the linear damping rate $\gamma_{d}$ and drive $\gamma_{L}$ have to be known very accurately in order to predict the correct non-linear behaviour (steady state, periodic, chaotic, bursting) [34] .

In this paper, the linear gyrokinetic code LIGKA[23, 18] is employed to describe the linear properties and to compare with the experiment. Since the equations had to be extended with respect to previous formulations [35] due to the inclusion of the NBI drive, a review of the equations is carried out here. In the following, we expand all Bessel functions (and Gamma functions for the background) due to the gyro-averaging operators and include all terms up to the fourth order in $k_{\perp} \varrho_{f}$ (with $k_{\perp}$ the perpendicular wave vector and $\varrho_{f}$ the fast particle gyro-radius), since even for energetic NBI particles with $E=93 \mathrm{keV}, k_{\perp} \varrho_{f}$ is less than 0.55. Here, the upper limits $\varrho_{f}=\frac{m_{f} v_{\perp}}{e_{f} B} \leq 3 \mathrm{~cm}$ 

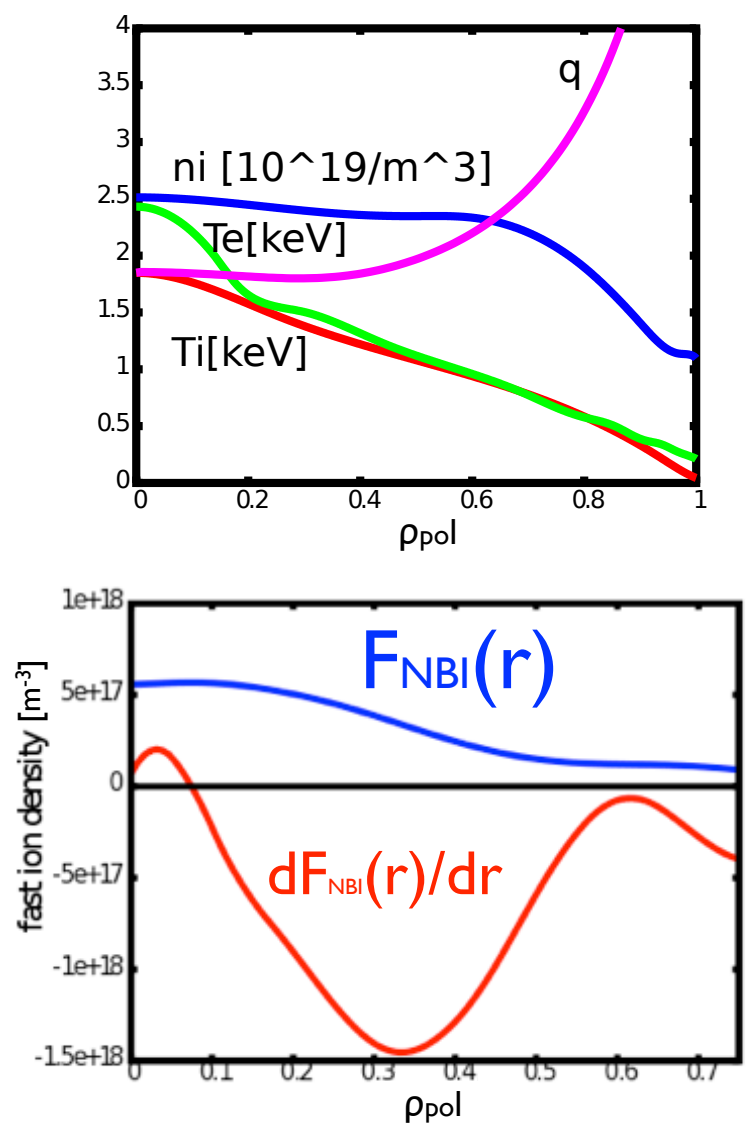
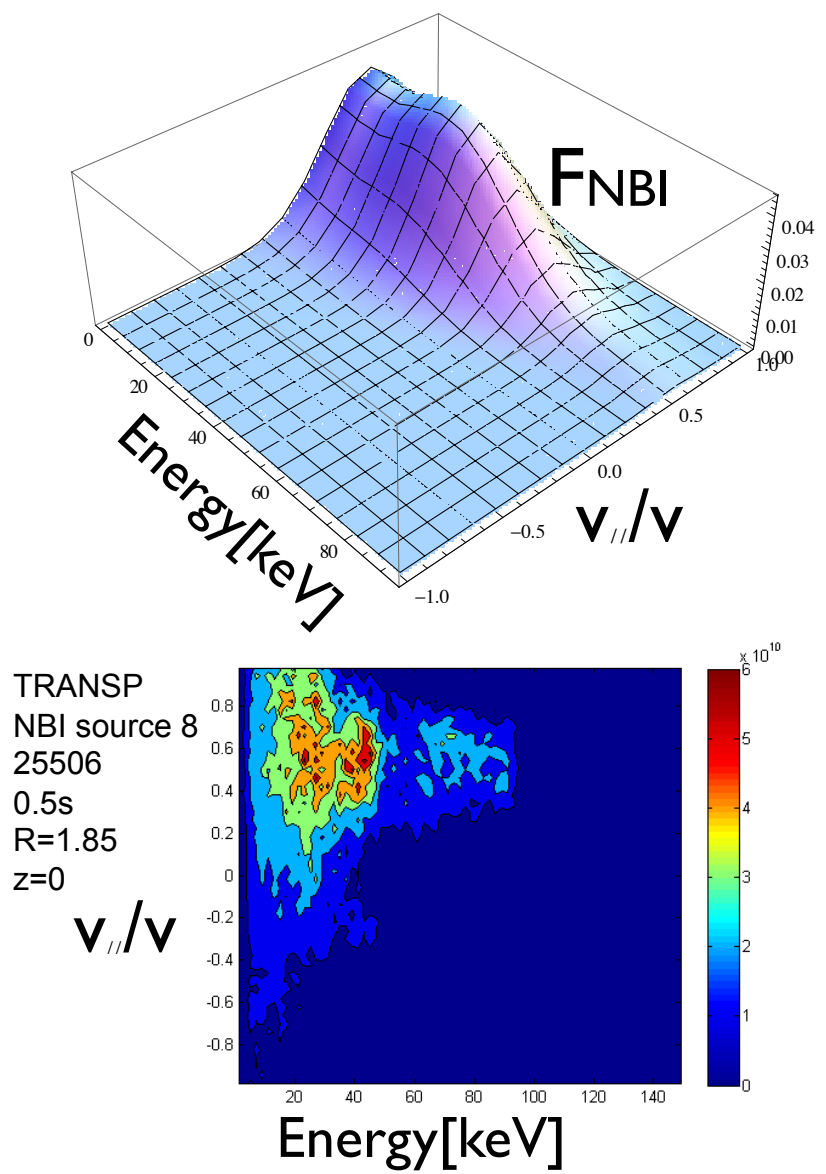

Figure 3. plasma background profiles (top left), radial fast particle profiles (bottom left) and the anisotropic distribution function as given by TRANSP (bottom right) and the fit used for the LIGKA simulations (top right)

and $k_{\perp}=\sqrt{k_{r}^{2}+k_{\theta}^{2}}=<18.5 m^{-1}$ were used with $k_{\theta}=-m / r$. For this estimate, the highest mode number $m=4$ and the innermost mode position $r=0.26 \mathrm{~m}$, and therefore $k_{\theta}=15.4 / m$ was used. $k_{r}$ was estimated based on the experimental data or 'a posteriori' from the LIGKA eigenfunctions via $k_{r} \approx 1 / \Delta r_{\text {mode }} \approx 1 / 0.1 m=10 / m$. Here $\Delta r$ is the radial mode width. The relative error $\mathcal{E}$ truncating $1-J_{0}^{2}(x)$ with $x=k_{\perp} \varrho_{f}$ at second order $\left(x^{2} / 2\right)$, i.e. $\mathcal{E}=\left(1-J_{0}^{2}(x)-\left(x^{2} / 2\right)\right) /\left(1-J_{0}^{2}(x)\right)$ is for the largest possible $k_{\perp} \varrho_{f}=0.55$ less than $6 \%$ and for truncating at fourth order $\left(x^{2} / 2-3 / 32 x^{4}\right)$ less than $0.3 \%$. Note that we assumed for this estimate that all the energy of the NBI particles is in the perpendicular motion, which is definitely not true for the particles in resonance with the mode. It will be shown below that these particles are mostly circulating and therefore have large parallel energy. Consequently, the truncation error is much smaller in this situation. The quasi-neutrality equation $(\mathrm{QN})$ is:

$$
0=\sum_{a} e_{a} n_{a 1}(\mathbf{x})=\sum_{a} e_{a} \int d^{2} \mathbf{v}\left(J_{0}\left(\varrho \nabla_{\perp}\right) f\right)_{a}
$$




$$
+\sum_{a} \nabla_{\perp} \frac{m_{a} n_{a 0}}{B^{2}} \nabla_{\perp} \phi(\mathbf{x})+\sum_{a} \frac{3 P_{a \perp}}{4 B^{2} \Omega_{a}^{2}} \nabla_{\perp}^{4} \phi(\mathbf{x})
$$

Here, the index $a$ counts the particles species, $e_{a}$ is the electrical charge, $m_{a}$ the particles' mass, $n_{a 1}$ is the perturbed density, $f_{a}$ the perturbed particle distribution function, $\Omega_{a}$ the cyclotron frequency $e_{a} B / m_{a}, P_{a \perp}$ the perpendicular pressure and $\phi(\mathbf{x})$ the perturbed electrostatic potential. Note, that the integration over the gyro-phase $\left(d^{3} v \rightarrow d^{2} v\right)$ has already been carried out.

Due to the low density of the energetic particles and the small 4th-order contribution, the energetic particles are neglected in the QN.

The gyrokinetic moment equation (GKM) reads:

$$
\begin{aligned}
& -\frac{\partial}{\partial t}\left[\nabla \frac{1}{v_{A}^{2}} \nabla_{\perp} \phi\right]+\mathbf{B} \cdot \nabla \frac{(\nabla \times \nabla \times A)_{\|} \cdot \mathbf{B}}{B^{2}}+\left(\mathbf{b} \times \nabla A_{\|}\right) \cdot \nabla \frac{\mu_{0} j_{0 \|}}{B}= \\
& -\sum_{a} \mu_{0} \int d^{2} \mathbf{v} e_{a}\left(\mathbf{v}_{d} \cdot \nabla J_{0} f\right)_{a}+\sum_{a} \mathbf{b} \times \nabla\left(\frac{\beta_{a \perp}}{2 \Omega_{a}}\right) \cdot \nabla \nabla_{\perp}^{2} \phi \\
& +\sum_{a} \frac{3 \beta_{a \perp}}{8 \Omega_{a}^{2}} \nabla_{\perp}^{4} \frac{\partial}{\partial t} \phi+\mathbf{B} \cdot \nabla \frac{1}{B} \sum_{a} \frac{\beta_{a}}{4} \nabla_{\perp}^{2} A_{\|}
\end{aligned}
$$

Here, $v_{A}$ is the Alfvén velocity, $A(\mathbf{x})$ the vector potential, $j_{0 \|}$ the parallel equilibrium current, $v_{d}$ the particles' drift velocity and $\beta_{a \perp}=2 \mu_{0} P_{a \perp} / B^{2}$. The term $\mathbf{b} \times \nabla\left(\frac{\beta_{\perp}}{2 \Omega}\right)$. $\nabla \nabla_{\perp}^{2} \phi$ can be rewritten for the background ions to be $\frac{i \omega_{* p}}{v_{A}^{2}} \nabla_{\perp}^{2} \phi$ with $\omega_{* p}=\mathbf{b} \times \frac{\nabla(n T)}{i e n B} \cdot \nabla$. It vanishes for electrons due to their small mass, but there can be a contribution from the EPs if their spatial pressure gradient is comparable to that of the background ions. However, one has to keep in mind that the gyroradius expansion discussed above is only valid for $k_{\perp} \varrho<1$ and therefore, in general, the full Bessel operator $1-J_{0}^{2}$ has to be retained for EPs. This term is the source for the diamagnetic correction of the first term on the lhs of the GKM equation, whereas the term $\nabla_{\perp}^{4} \phi$ leads to the kinxetic Alfvén wave correction. The last term on the rhs of the GKM equation can be interpreted as a finite- $\beta$ correction to the second term of the lhs. Since it does not contain perpendicular gradients of $\beta_{a}$, this correction is small, also for energetic particles.

The perturbed distribution function $f$ is governed by the gyrokinetic equation. After splitting off the adiabatic part by introducing

$$
f=h+H_{1} \frac{\partial F_{0}}{\partial E}-\left[e \frac{\partial F_{0}}{\partial E}-\frac{c \nabla F_{0}}{i \omega B} \cdot(\mathbf{b} \times \nabla)\right] J_{0} \psi(\mathbf{x})
$$

with $\psi$ defined by $A_{\|}=(\nabla \psi(\mathbf{x}))_{\|} /(i \omega), H_{1}=e J_{0} \phi-e J_{0} U A_{\|}$and $U$ the parallel gyrocentre velocity, the integrals in the QN and GKM equation can be written in the following form:

$$
\left(\int J_{0} h d^{2} \mathbf{v}\right)^{c i r c}=-\frac{\pi}{2} e_{a} v_{t h}^{3} \sum_{m} \int_{0}^{\Lambda_{\min }(r)} d \Lambda \int_{0}^{\infty} d Y \sqrt{Y} \cdot \sum_{k} \sum_{\sigma} \frac{\partial F_{0}}{\partial E}
$$




$$
\begin{aligned}
& \frac{\left(\omega-\hat{\omega}_{*}\right) K_{m, p, k}}{\omega-\omega_{D}-\left(\sigma S_{m}+k\right) \omega_{t}} \cdot J_{0}^{2}\left[a_{k, m, \sigma} \phi_{m}(r)-\left(a_{k, m, \sigma}-a_{k, m, \sigma}^{G}\right) \psi_{m}(r)\right] \\
& \left(\int J_{0} h d^{2} \mathbf{v}\right)^{\text {trapped }}=-\frac{\pi}{2} e_{a} v_{t h}^{3} \sum_{m} \int_{\Lambda_{\min }(r)}^{\Lambda_{\max }(r)} d \Lambda \int_{0}^{\infty} d Y \sqrt{Y} \cdot \sum_{k} \frac{\partial F_{0}}{\partial E} \\
& \frac{\left(\omega-\hat{\omega}_{*}\right) K_{m, p, k}}{\omega-\omega_{D}-k \omega_{t}} \cdot J_{0}^{2}\left[a_{k, m} \phi_{m}(r)-\left(a_{k, m}-a_{k, m}^{G}\right) \psi_{m}(r)\right]
\end{aligned}
$$

Here, $\Lambda=\mu B_{0} / E$ with $\mu$ the magnetic moment, $B_{0}$ is the on-axis magnetic field, $\Lambda_{\min }$ and $\Lambda_{\max }$ the minimal and maximal values of $\Lambda$ for passing and trapped particles, $E$ is the energy variable, $Y=E / T, v_{t h}=\sqrt{2 T / m}, S_{m}=n q-m$ and $\hat{\omega}_{*}=-\frac{\mathbf{b} \times \nabla F_{0}}{i e B \partial F_{0} / \partial E}$. The summation over co- and counter-passing particles is indicated by the sign variable $\sigma= \pm 1$.

The advantage of rewriting the equations in terms of $\psi$ instead of $\mathbf{A}_{\|}$is that the (small) perturbed electric field responsible for damping and drive can be expressed by the difference of $\phi$ and $\psi$, more precisely $E_{\|}=-(\nabla \phi)_{\|}-\partial A_{\|} / \partial t=-\nabla(\phi-\psi)_{\|}=-k_{\|}(\phi-\psi)$. For stable Alfvén waves with negligible damping no electric field is present and $\phi=\psi$. This is the reduced MHD limit of the GKM equation shown above. At lower frequencies the Alfvén waves couple to the electrostatic/acoustic waves and $\phi \neq \psi$. Therefore, the difference $\phi-\psi$ is a signature of the polarisation of a wave and its radial envelope allows to identify the radial location of damping and drive.

These integrals of equations 2 and 3 are solved numerically. The transit/bounce frequencies $\omega_{t, b}$, the precession frequency $\omega_{D}$ and the orbit-integrated coefficients $a_{k, m}$, $a_{k, m}^{G}$ and $K_{m, p, k}$ (given in the appendix) are calculated accurately using the HAGIS [33] code. For the denominator a rational interpolation scheme is used to allow for an accurate calculation of the residuum in case of damped modes, i.e. $\gamma<0$.

It can be shown [35] that this set of equations can be simplified to the reduced MHD equations and therefore the influence of kinetic extensions can be traced. The structure of the equations is a second order integro-differential equation. As in reduced MHD, the (local) continuous spectrum can be determined by finding the zeros of the coefficient of the second order term. Fourth order terms are neglected for this local analysis. It should be noted that also EPs can contribute to this second order term: both $\frac{i \omega_{* p}}{v_{A}^{2}} \nabla_{\perp}^{2} \phi$ and $\int d^{3} \mathbf{v}\left(e \mathbf{v}_{d} \cdot \nabla J_{0} f\right)_{E P, \text { radial }}$ are proportional to $\beta_{E P}$ and therefore modify the real and the imaginary part of the second order coefficient.

The relevant dispersion relation was re-derived [18] for the gyrokinetic model [35] underlying the eigenvalue code LIGKA. As can be seen in equations 2 and 3, the integration over velocity space is carried out completely numerically, and therefore it is possible to include a rather general distribution function for the EPs. Based on a TRANSP simulation [32] (see fig 3), a parameterised fit is generated, ensuring that the distribution is smooth in energy $E$ and pitch angle $\lambda=v_{\|} / v$. Although this fit does not reproduce the small-scale details of the TRANSP simulation, it is a reasonable approximation for the anisotropy in $\lambda$ and the energy dependence of the width of $F_{E P}$ in the $\lambda$ direction. 


\section{Numerical results}

Using the CLISTE code [36], a sequence of equilibria for \#25506 between $t=0.35$ and $t=0.55$ was reconstructed. The on-axis EP pressure is, based on the equilibrium reconstruction and the TRANSP simulations, $\beta(r=0)=0.7 \%$. The other kinetic profiles are shown in fig 3 . The analysis at $t=0.35 \mathrm{~s}$ for the RSAEs and its radial harmonics is carried in ref [7]. One of the main conclusions of that paper was that the q-profile in the centre is only very slightly reversed, i.e. it is basically almost flat. Therefore, also at $t=0.45 \mathrm{~s}$ a flat q-profile was assumed (see fig 3 ). The corresponding kinetic continuum (in the sense of the equations of the last section) can be seen in fig 4. Both the solution with and without trapped background ions are plotted. As shown previously [31, 18], trapped particles cause a downshift of the local spectrum. The minimum in the continuum is caused by the geodesic coupling. Since at $q=2$ the sideband corrections $\left(1 / q^{2}\right)$ are not very large, the simple dispersion relation

$\omega_{B A E}^{2}=\frac{v_{t h}^{2}}{R_{0}^{2}}\left[\frac{7}{4}+T_{e} / T_{i}\right]$ is approximately valid and therefore $\omega_{B A E}$ is approximately constant as $q$ varies. This behaviour is clearly seen also experimentally (see fig 1). One expects a small increase of $\omega_{B A E}$ due to the rising temperature during the time window under consideration, however this effect is cancelled (by coincidence) by the movement of the mode radially outwards as can be seen experimentally and from the local analysis (see ref [7], fig 3). Including the measured toroidal rotation $8 \mathrm{kHz}$, the minimum frequency is found to be $54 \mathrm{kHz}$, if trapped background ions are included.

For $n=1, m=2$, the inclusion of energetic ions results in a small up-shift of the local continuum whereas for $n=2, m=4$, an offset of $15 \mathrm{kHz}$ is found (see fig 4). As mentioned in the previous section, this up-shift is due to the $\omega_{* p}$ contribution of the EPs that depends on the poloidal mode number $m$. It has to be noted that for this local analysis the orbit widths of the EPs is neglected. That means that their contribution is overestimated. But since the bulk of the EPs is mainly passing (thin radial orbits) this error is not very large. Adding $n \cdot 8 \mathrm{kHz}$ of rotation, one finds a frequency separation of $17 \mathrm{kHz}$ between the $n=2$ mode $(75 \mathrm{kHz})$ and the $n=1$ mode $(58 \mathrm{kHz})$ that is close to the experimentally observed value of $20 \mathrm{kHz}$ (see fig 2 ).

In fig 5 the global solution for the $n=1$ case is plotted. The mode exists just below the minimum of the continuum $f=53.5 \mathrm{kHz}$, even without energetic particles. At a later time-point, when the magnetic shear at the minimum of the continuum accumulation point increases (all other parameters are kept constant), no global solution can be found. This demonstrates that only weak shear $s=r q^{\prime} / q<0.8$ allows the mode to exist and it disappears when it moves radially outwards. The damping $\gamma / \omega=-2.0 \%$ is mainly radiative damping and some ion Landau damping, as can be seen when the radial electric field is plotted (see fig 5). However, since $\phi$ and $\psi$ are almost equal, the polarisation of the mode is mainly Alfvénic.

Comparing this mode to the experimental ECE mode structure (fig. 2) one finds that the radial mode width as calculated with LIGKA (w/o EPs) is slightly smaller.

Now the drive mechanism is investigated. Analysing the resonance condition $\omega-n \omega_{\text {prec }}-$ 

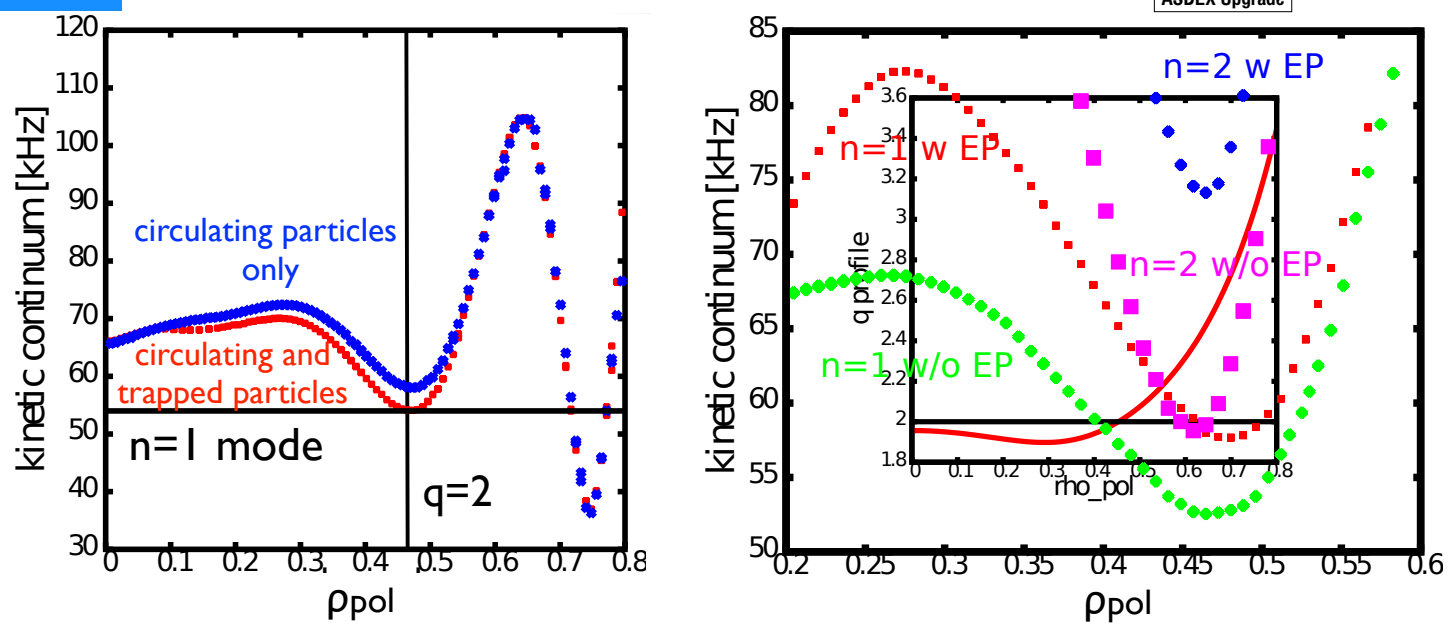

Figure 4. local kinetic continuum for 25506 at $0.47 s$ : left the effect of trapped background particles; right: the inclusion of EPs in a local approximation shifts the spectra
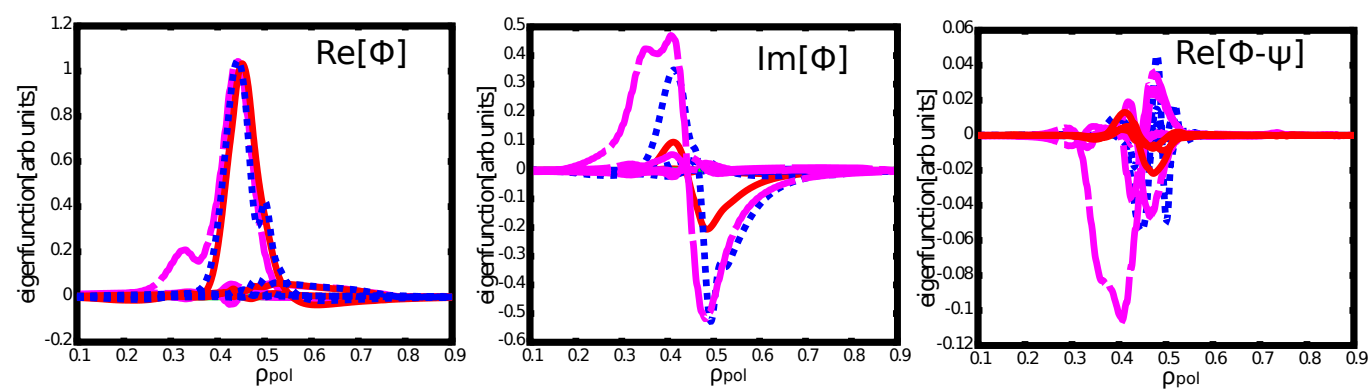

Figure 5. mode structures $(n=1)$ : real part of the electrostatic potential $\phi$ (left), imaginary part i.e. phase shift of $\phi$ (middle) and electric field $\phi-\psi \sim E_{\|}$(right). The stable BAE is shown (solid red) as well as the unstable BAE with an isotropic distribution function (dotted blue) and the NBI-driven BAE (dashed magenta). Note that the real part of the eigenfunctions is scaled to unity, whereas the imaginary part and the difference $\phi-\psi \sim E_{\|}$do not contain further scaling factors. Since $\phi-\psi$ is much smaller than $\phi$ or $\psi$ itself, the mode is called mainly Alfvénic.

$(n q-m+k) \omega_{t}=0$ at $\varrho_{p o l}=0.45$, one finds (see fig 6$)$ that the principle resonances occur for the fundamental harmonics $(k=1,2)$ of the transit frequency $\omega_{t}$. The precessional resonances do not play a significant role since $\omega_{\text {prec }} \ll \omega$ in the whole velocity space and in particular for passing NBI ions (see fig. 7). The fraction of trapped NBI ions is very small at $\varrho_{p o l}=0.45$ and is therefore not plotted in fig. 6. The NBI destabilisation mechanism can be directly analysed, if the expressions (2) and (3) that are proportional to $\delta W$ are plotted as a function of $\varrho_{p o l}$. In fig. 6 , the sum of the circulating and trapped coefficients (eqn 2 and 3) for $\phi$ are plotted, neglecting the radial structure of $\phi$ in order to demonstrate the effect of the EP distribution function alone: both the isotropic and the NBI distribution function of fig. 3 (right) have a positive, i.e. destabilising imaginary part. The total $\beta_{\text {fast }}$ was kept constant and only the distribution function in 

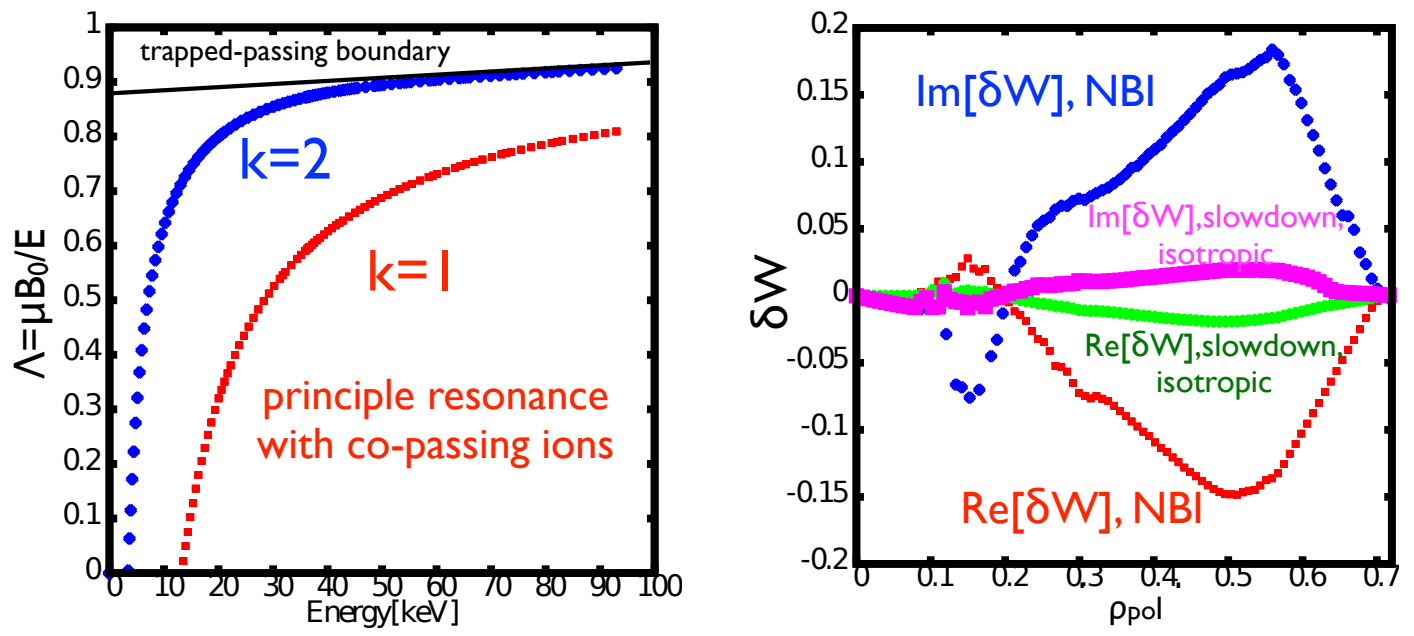

Figure 6. the resonance condition $\omega-n \omega_{\text {prec }}-(n q-m+k) \omega_{t}=0$ at $\varrho_{\text {pol }}=0.45$ for circulating ions in $\Lambda-E$ phase space (left) and the contribution of the EPs to the overall $\delta W$ (details in the text)
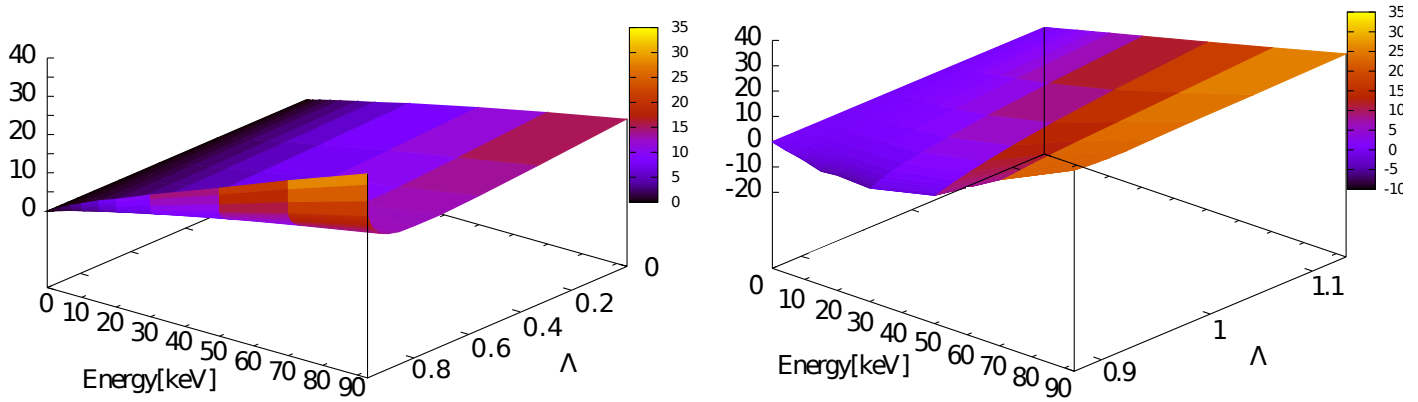

Figure 7. precession frequency of passing and trapped deuterium ions (NBI) at $\varrho_{\text {pol }}=0.45:$ the frequencies are too low $(\max 35 \mathrm{kHz})$ to drive the observed BAE modes via the precessional resonance

the coordinate $\lambda$ was changed. For the NBI case, the drive is much stronger since there is a maximum in the distribution function at the main resonances around $\lambda=0.6$ and therefore $\Lambda=\left(1-\lambda^{2}\right) * B_{0} / B \approx 0.7$.

The influence of the EPs on the $n=1$ mode structure is shown in figure 5 : the radial mode structure becomes slightly broader (left) and the phase shift (middle) increases (anti-symmetrically) around the mode peak location. The parallel electric field that is a measure for the damping (w/o EPs) or a measure for the mode drive i.e. the inverse Landau damping ( $\mathrm{w}$ EPs) is shown on the right of fig. 5. The damping occurs at the position of the mode and is therefore local. Consequently, the damping must be a combination of radiative damping (the mode is close to the continuum) and ion Landau damping ( $\omega \sim 1,2, \ldots \cdot \omega_{\text {transit }}$ for background ions in the Maxwell tail). The continuum damping at $\varrho_{\text {pol }}=0.7$ is very small.The drive with a different sign than the damping occurs also locally but is maximal in the steepest gradient region $\left(\varrho_{p o l}=0.35\right)$ as shown in fig. 3. 

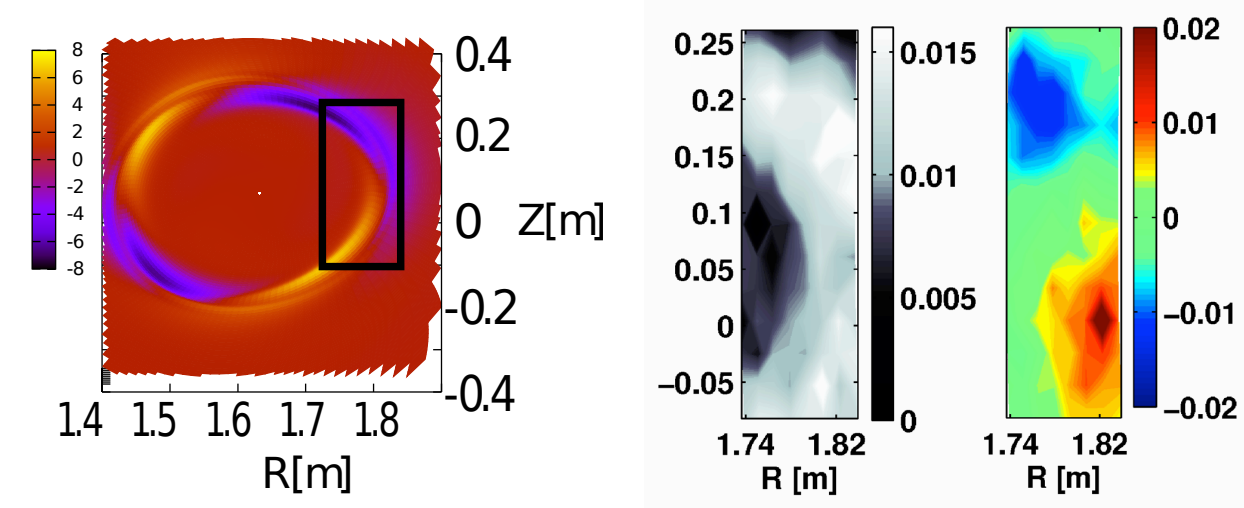

Figure 8. $2 \mathrm{~d}$ mode structures $(n=1)$ : on the left LIGKA $\left(\phi \sim \delta T_{e}\right)$, on the right the ECEI data (see also fig 2)

The damped mode $\gamma / \omega=-2.0 \%$ becomes unstable, $\gamma / \omega=0.9 \%$ for an isotropic distribution function and $\gamma / \omega=2.1 \%$ for the NBI distribution function. The real mode frequency shifts from $53 \mathrm{kHz}$ to $54 \mathrm{kHz}$ (isotropic) and $55.5 \mathrm{kHz}(\mathrm{NBI})$. The $2 \mathrm{~d}$ mode structure of the anisotropic NBI driven mode (dashed magenta in figure 5) is very similar to the experimental one (fig. 8, right).

The $n=2$ mode can only be found in the anisotropic case at a frequency of $76 \mathrm{kHz}$, i.e. in the kinetic continuum. As can be seen experimentally (fig. 2) the mode stops to exist after $t=0.47 \mathrm{~s}$ i.e. at $t=0.45 \mathrm{~s}$ the mode existence criteria become already marginal and therefore no detailed investigation as for the $n=1$ mode could be carried out.

\section{Conclusions}

In plasmas with weak reversed shear, electromagnetic NBI-driven modes at the BAE/GAM frequency were observed at ASDEX Upgrade. Using a linear gyrokinetic model the local continuum and the global self-consistent eigenfunctions were determined. Both, the mode frequencies and the mode structure are in reasonable agreement with the experimental findings. The existence of global modes below the minimum of the kinetic continuum was demonstrated for small shear.

The observed modes cannot be precessional fishbones or double tearing modes for the following reasons: 1 . The precessional frequency of the EPs is not large enough to drive the mode at that large frequency $(60 \mathrm{kHz}) .2$. The mode structures are rings moving radially outwards. 3 . The frequencies of the $n=1$ and $n=2$ modes are very similar and are not higher harmonics (as it would be the case for fishbones). 4. In other discharges [37] transitions from steady state to bursting and vice versa were observed.

A very important result of this paper is that the mode structure of the bursting modes does not seem to be substantially different from the linear gyrokinetic solution. Although further cases with different mode numbers and different EP drive will have to be analysed, so far, both the radial envelope and the phase shift match quite accurately. 


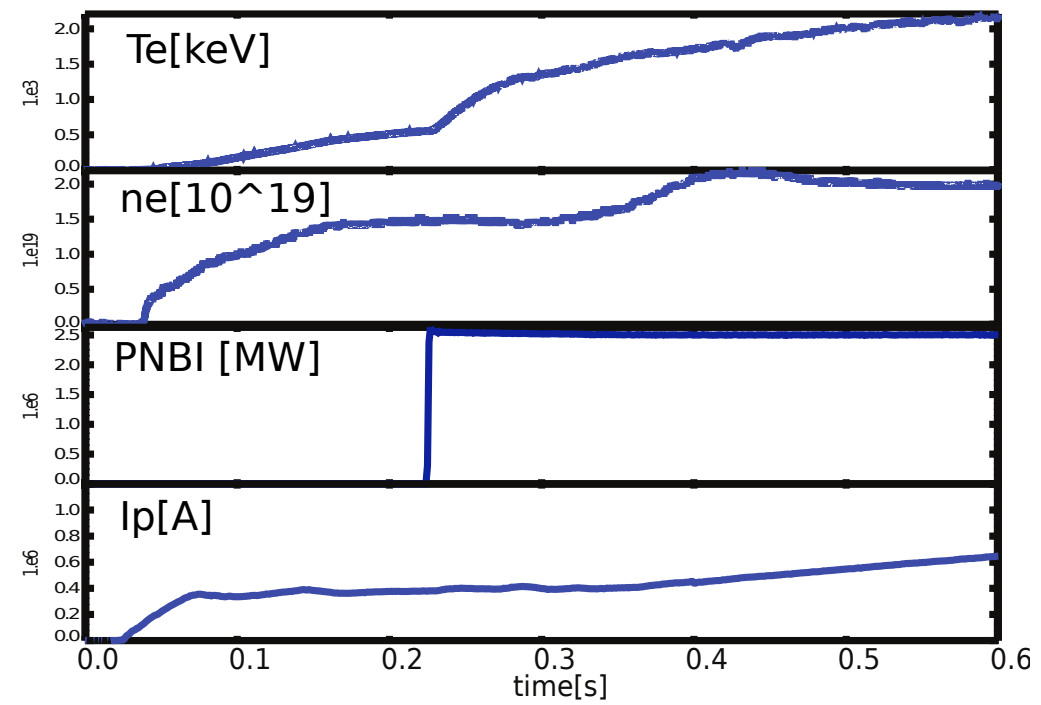

Figure 9. time traces of \#25506 for central $T_{e}$, line averaged $n_{e}$, NBI power and the current ramp $I_{p}$.

This gives some confidence that non-linear hybrid models with a slowly evolving mode structure but full EP dynamics in velocity space will be able to capture the main features of non-linear EP transport.

Another important future research area is the role of these modes in reversed shear scenarios at ITER (scenario 4): since the BAEs are located closer to the plasma edge than RSAEs they might be more efficient in expelling EPs (as it is seen at ASDEX Upgrade), especially in the non-linear bursting phase.

\section{Appendix}

$$
\begin{aligned}
& a_{m, k}=\frac{1}{\tau_{t}} \int_{-\tau_{t} / 2}^{\tau_{t} / 2} d \hat{t} e^{i\left[S_{m} \theta-\left(k+S_{m}\right) \omega_{t} \hat{t}\right]} \\
& a_{k m}^{G}=\frac{1}{\tau_{b, t}} \int_{-\tau_{b, t} / 2}^{\tau_{b, t} / 2} d \hat{t} \frac{\omega_{d}(r, \theta)}{\bar{\omega}_{d}(r)} e^{i\left[S_{m} \theta-\left(k+S_{m}\right) \omega_{t} \hat{t}\right]} \\
& K_{m, p, k}=\frac{1}{2 \pi} \int_{-\pi}^{\pi} \frac{d \theta}{b(r, \theta) \sqrt{1-\Lambda / b(r, \theta)}} e^{-i\left[S_{p} \theta-\left(k+S_{m}\right) \omega_{t} \hat{t}(\theta)\right]}
\end{aligned}
$$

where $\hat{t}$ is the time since the particle passed through a reference point on its orbit e.g. the outboard midplane. Furthermore, $b(r, \theta)=B_{0} / B(r, \theta), S_{p}=n q-p$ and $\bar{\omega}_{D}$ is the orbit averaged precession drift. Here, $p$ is the 'mode' number of the projection operator $e^{i p \theta}$ that is needed to construct the weak form of the integral expressions. For further details refer to reference [23]. 
[1] ITER Physics Expert Group on Energetic Particles, Heating and Current Drive et al. 1999 Nucl. Fusion 392471

[2] Goeldbloed J.P. et al., Plasma Physics Controlled Fusion 35 (1993) B277

[3] Sharapov S.E. et al., Phys. Letters A 289 (2001) 127

[4] Fasoli A. et al., Plasma Physics Control. Fusion 44 (2002) B159

[5] Berk HL, Borba DN,Breizman BN, Pinches SD and Sharapov SE 2001 Phys. Rev. Lett. 87185002

[6] Tobias BJ, Classen IGJ, Domier CW, Heidbrink WW, Luhmann Jr. NC, Nazikian R, Park HK, Spong DA and Van Zeeland MA 2011 Phys. Rev. Lett. 10675003

[7] I.G.J. Classen et al, Plasma Phys. Control. Fusion 53 (2011) 124018

[8] Ph. Lauber, S. Günter, S.D. Pinches,"Damping rates of global kinetic modes in tokamaks", Proceedings of the 3rd IAEA technical meeting on Theory of plasma instabilities (CDROM), York (2007)

[9] N. Gorelenkov et al, Plasma Phys. Control. Fusion 48 (2006) 12551269

[10] M Brüdgam, PhD Thesis: 'Nonlinear Effects of Energetic Particle Driven Instabilities in Tokamaks', TUM München, 2010 (http://mediatum2.ub.tum.de/node?id=958179)

[11] A.D. Turnbull et al Phys. Fluids B 5, 2546 (1993)

[12] B. Heidbrink et al, Phys. Rev. Letters 71 (1993)

[13] Mikhailovski et al, Nucl Fusion 13259 (1973)

[14] B. Coppi and F. Porcelli, Phys. Rev. Lett. 57, 2272 (1986).

[15] F. Porcelli, Plasma Phys. Control. Fusion 33, 1601 (1991)

[16] Chu M S et al Phys Fluids B 4 3713(1992)

[17] F. Zonca et al, Plasma Phys. Control. Fusion 38, 2011 (1996)

[18] Ph Lauber et al 2009 Plasma Phys. Control. Fusion 51124009

[19] H.S.Zhang, Z.Lin, I.Holod, X. Wang, Y.Xiao and W.L.Zhang, Phys.Plasmas 17, 112505 (2010)

[20] C. J. Boswell et al., Phys. Lett. A 358, 154 (2006)

[21] H. L. Berk et al., Nucl. Fusion 46, S888 (2006)

[22] R. Nazikian et al., Phys. Rev. Lett. 101,185001 (2008)

[23] Ph. Lauber et al, J. Comp. Phys., 226/1 (2007)

[24] M.Garcia-Munoz et al. in Fusion Energy 2008 (Proc. 22nd Int. Conf. Geneva, 2008) (Vienna: IAEA) CD-ROM file [EXW/P7-07] and http://wwwnaweb.iaea.org/napc/physics/FEC/FEC2008/html/index.htm

[25] M Garcia-Munoz et al. 2011 Fast Ion Transport Induced by Alfvn Eigenmodes in the ASDEX Upgrade Tokamak submitted to Nucl. Fusion

[26] Van Zeeland MA et al. 2011 Physics of Plasmas 18056114

[27] V. Igochine et al, Nucl. Fusion 43, 1801 (2003)

[28] Classen IGJ et al. 2010 Rev. Sci. Instrum. 81 10D929

[29] M Garcia-Munoz, Fahrbach H-U and Zohm H 2009 Rev. Sci. Instrum. 80053503

[30] M Garcia-Munoz, et al, Phys. Rev. Letters 100, 055005 (2008)

[31] I Chavdarovski and F Zonca, Plasma Phys. Control. Fusion 51, 124009 (2009)

[32] B. Geiger et al, Plasma Phys. Control. Fusion 53065010 (2011)

[33] S.D. Pinches, Comp. Phys. Comm., 111 (1998)

[34] Berk HL, Breizman BN and Pekker M 1996 Phys. Rev. Lett. 76 1256-9

[35] H. Qin et al, Phys. Plasmas 5, 1035 (1998)

[36] P. Mc Carthy, Plasma Phys. Control. Fusion 54, 015010 (2012)

[37] Ph. Lauber et al,"NBI driven Alfvnic Modes at ASDEX Upgrade" presented at the 12th IAEA TCM, Austin, Texas, Sept 7 - 10 (2011), http://w3fusion.ph.utexas.edu/ifs/iaeaep/talks/s5-i5lauber-philipp-ep-talk.pdf 